\title{
Genetic relationship and maturity state of the allorecognition system affect contact reactions in juvenile Seriatopora corals
}

\author{
Yoko Nozawa ${ }^{1,3}$, Yossi Loya ${ }^{2, *}$ \\ ${ }^{1}$ Sesoko Station, Tropical Biosphere Research Center, University of the Ryukyus, Sesoko, Motobu, Okinawa 905-0227, Japan \\ ${ }^{2}$ Department of Zoology, Tel Aviv University, George S. Wise Faculty of Life Sciences, Tel Aviv, 69978, Israel \\ ${ }^{3}$ Present address: Amakusa Marine Biological Laboratory, Kyushu University, Tomioka, Reihoku-Amakusa, \\ Kumamoto 863-2507, Japan
}

\begin{abstract}
Adult stony corals are characterized by a well-developed allorecognition system. In most cases, genetically identical coral grafts fuse, whereas genetically different grafts (allografts and xenografts) show various histoincompatible responses. Very little is known, however, about the allorecognition system in juvenile corals. We studied contact reactions of juvenile pairs in 2 scleractinian coral species: Seriatopora caliendrum and S. hystrix (0 to $59 \mathrm{~d}$ after settlement). Juvenile colonies were experimentally grafted in combination groups of various genetic distances: sibling allografts of $S$. hystrix (4 pairs), non sibling allografts of $S$. caliendrum (12 pairs), non sibling allografts of $S$. hystrix (16 pairs) and xenografts of the 2 species (26 pairs). Three tissue contact reactions were detected in all of the genetic combination groups: nonfusion, fusion and incompatible fusion. The transition in contact reaction patterns was recorded over a $1 \mathrm{yr}$ period. Contact reactions and their duration were largely consistent among pair replicates of each combination and within each of the 4 genetic combination groups. We suggest that in juvenile Seriatopora corals, the genetic combination of each graft is a primary determinant of the contact reaction and its endurance. Maturity state of the allorecognition system, affects both onset and duration of the fusion and incompatible fusion reactions in the juvenile corals. Onset of the fusion reaction was observed only when grafted colonies were younger than $4 \mathrm{mo}$. Throughout the experimental period, most fusion reactions transformed into histoincompatible reactions or disconnection and virtually none of the allografts or xenografts maintained stable chimeras. We suggest that as the alloimmune system matures, incompatible fusion reactions eventually transform into nonfusion or disconnection via tissue necrosis of the contact zone.
\end{abstract}

KEY WORDS: Corals · Allorecognition · Contact reactions $\cdot$ Allografts $\cdot$ Xenografts $\cdot$ Seriatopora spp.

\section{INTRODUCTION}

For marine colonial invertebrates, an accurate allorecognition system is essential, since direct tissue to tissue contact with neighbour colonies is inevitable as they grow. Hence, many of these taxa have been studied with respect to their allorecognition capabilities (Grosberg 1988, Leddy \& Green 1991).

The existence of an allorecognition system is welldocumented in adult coral species: genetically identical grafts (autografts) fuse, whereas genetically differ- ent grafts (allografts and xenografts) rarely fuse and show various histoincompatible responses (Chornesky 1991, Chadwick-Furman \& Rinkevich 1994, Frank \& Rinkevich 1994, Jokiel \& Bigger 1994, Rinkevich et al. 1994, Frank et al. 1996).

In contrast to these allograft histoincompatible contact responses between adult corals, recurrent incidences of allogeneic fusion have been reported between juvenile corals (Hidaka 1985, Frank et al. 1997, Hidaka et al. 1997, Barki et al. 2002). Hidaka (1985) was the first to study contact reactions 
between juvenile corals (primary polyps) in the scleractinian coral Pocillopora damicornis. He found that all 22 allografts of the juvenile pairs fused, whereas no fusion was observed in allografts of their maternal colonies. Hence, he suggested that juvenile corals might lack the functional allorecognition system shown by adult corals. Hidaka et al. (1997) further confirmed the appearance of a fusion reaction in allografts of the juvenile coral $P$. damicornis and observed its stability over a 4 to 6 mo experimental period. In addition, they found 2 histoincompatible reactions in the allografts; nonfusion and a newly described type of contact reaction in coral species, which they termed 'incompatible fusion', characterized by fusion of tissues with few zooxanthellae at the interface zone. The initial contact responses were not affected by the time elapsed between planulation and initial contact (a maximum of $3 \mathrm{mo}$ ), and replicated pairs of each allograft combination showed similar contact reactions. Hence, they concluded that genetic composition might be the primary determinant of contact reactions in allografts of juvenile corals. Similarly, in a study of allografts between newly settled planulae of the scleractinian coral Stylophora pistillata, Frank et al. (1997) reported that allografts of juvenile corals had the same 3 contact reactions. They also observed that the fused allografts maintained stable chimeras throughout the 8 mo experimental period. However, in their experiment, the allografts established from colonies younger than 2 mo post settlement always fused, while those from colonies between 2 and 4 mo old initially fused, then disconnected via transient fusion (i.e. incompatible fusion sensu Hidaka et al. 1997), and allografts from colonies older than 4 mo always showed nonfusion. This led them to conclude, in contrast to Hidaka et al. (1997), that contact reactions of the allogeneic juvenile corals solely depend on the age of the grafted colonies. In 4 soft coral species, Barki et al. (2002) observed only 2 types of contact responses, fusion and nonfusion, occurring between allogenic primary polyps. Their follow-up observations (up to $450 \mathrm{~d}$ ) on the allogenic chimeras of the soft corals revealed that all of the chimeras had disconnected by the end of the experimental period.

These studies led us to address the following 3 questions: (1) Do the 3 contact reactions (fusion, nonfusion and incompatible fusion) constitute general responses between genetically different grafts of juvenile Pocilloporid corals? (2) Do fused allografts of juvenile Pocilloporid corals form and maintain stable chimeras or do they eventually separate as the alloimmune system matures, in a similar way to chimeras of juvenile soft corals? (3) What is the major determinant of contact reactions in juvenile corals; their genetic combination and/or the maturation state of their allorecognition systems?

To examine these questions, juvenile coral pairs of various genetic and age combinations of the Pocilloporid corals Seriatopora caliendrum and S. hystrix were experimentally brought into contact, and the transition in their contact reactions was followed over a 1 yr experimental period.

\section{MATERIALS AND METHODS}

Colonies of the brooding corals Seriatopora caliendrum and $S$. hystrix were collected just before the first quarter moon from Sesoko and Bise reefs in June, July and August 1997. The 2 reef sites are about $8 \mathrm{~km}$ apart in the northern part of Okinawa Island, Japan $\left(26^{\circ} \mathrm{N}\right.$, $127^{\circ} \mathrm{E}$ ). To avoid possible collection of colonies of the same genotype, resulting from fragmentation, collection of each colony was done at intervals $>100 \mathrm{~m}$ between colonies. The corals were transferred to Sesoko station, Tropical Biosphere Research Center, where each colony was numbered and maintained individually in a plastic bucket with running seawater. Planulae were collected by sieving running seawater, which overflowed from the bucket into a $500 \mathrm{ml}$ plastic container with 2 'windows' covered with $180 \mu \mathrm{m}$ mesh plankton net. The planulae collectors were set from dusk to dawn daily from June to August. Planulae released from individual colonies were collected separately each dawn and placed in Petri dishes filled with seawater. A new plastic sheet (i.e. biologically unconditioned) was placed on the bottom of each dish to serve as a settlement substrate. Most planulae settled on the sheets within a few hours after being introduced into the dishes. The settled planulae (all aged less than $24 \mathrm{~h}$ post planulation) were used for the grafting experiments. In order to establish coral pairs of different ages, some of the settled planulae were separated and maintained individually in aquaria with running seawater under indirect natural light.

To examine possible effects of genetic difference on contact reactions of juvenile corals, combinations of sibling allografts (i.e. pairs of juveniles originating from the same colony), non sibling allografts (i.e. pairs of settled planulae originated from different conspecific colonies) and xenografts (i.e. pairs of settled planulae originated from colonies of different species) were made using juvenile colonies of Seriatopora caliendrum and S. hystrix. However, the genetic origin of planula larvae in $S$. caliendrum and $S$. hystrix, as well as other Pocilloporid corals, has not been fully elucidated yet (i.e. sexual and/or asexual reproduction; see Permata et al. 2000). Hence, examination of the effect of genetic difference was performed assuming genetic 
similarity among sibling planulae. Assumed genetic differences among the combinations were: sibling allografts < non sibling allografts $<$ xenografts. To examine possible effect of age on contact reactions of juvenile corals, various age combination-pairs of young (within the first $7 \mathrm{~d}$ after settlement) and older juvenile colonies (aged 8 to $59 \mathrm{~d}$ after settlement) were also made in each genetic combination group.

Pairs of newly settled planulae (i.e. primary polyps) were brought into tissue contact using a modification of the method described by Hidaka et al. (1997). Two primary polyps that had settled on the same plastic sheet (for sibling allografts) or on separate plastic sheets (for non sibling allografts and for xenografts) were juxtaposed on a glass slide, by cutting the plastic sheet along the edges of the primary polyps. The

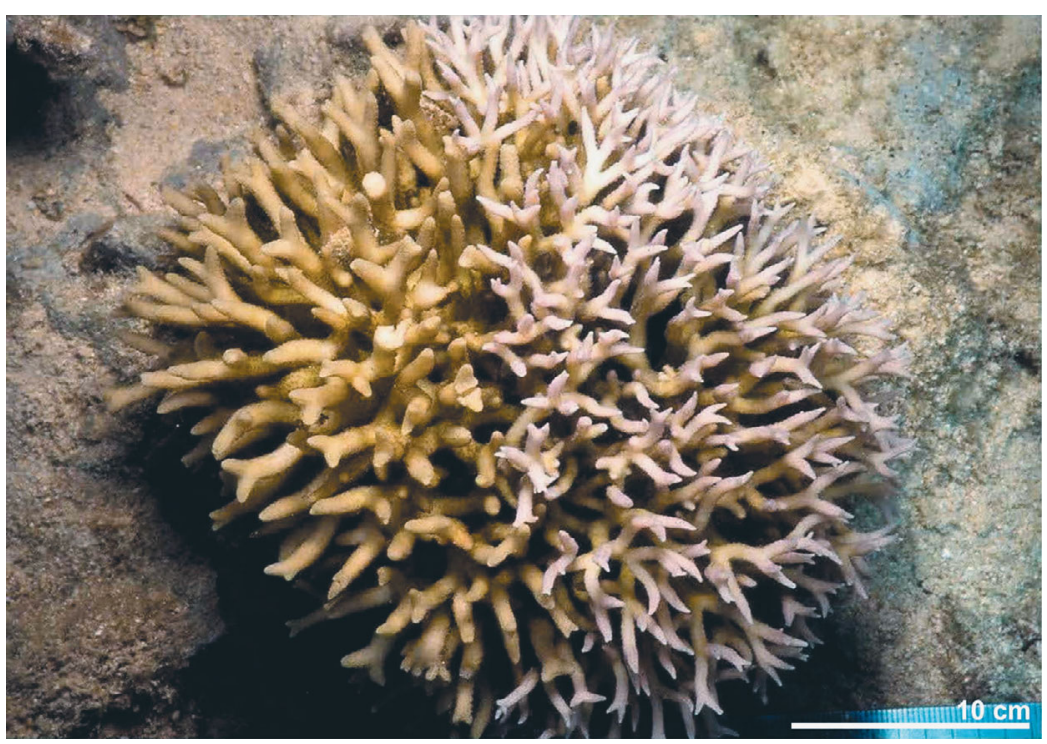

Fig. 1. Seriatopora caliendrum and S. hystrix: a naturally occurring xenograft of adult colonies of $S$. caliendrum (left) and of $S$. hystrix (right) on a reef near the Sesoko Marine Laboratory sheets were then fixed with a pair of clamps comprising silicon tubes glued to both sides of the glass slide and 2 plastic straps. The pairs were numbered and maintained in a tank supplied with running seawater under natural light at the Sesoko station for a $1 \mathrm{yr}$ experimental period.

Of the over 100 juvenile coral pairs originally prepared for this experiment, only 58 pairs were examined, due to mortality or detachment of one or both juvenile colonies, within the first few weeks prior to the establishment of any contact reaction (see Table 1 for the number of pairs in each combination group). The coral pairs were observed and photographed weekly under a dissecting microscope for the first $6 \mathrm{mo}$ and fortnightly for the next 6 mo. The glass slides on which the coral pairs were affixed were periodically cleaned with a fine paintbrush.

Seven naturally occurring xenografts of adult colonies (ca. 20 to $30 \mathrm{~cm}$ in diameter) of Seriatopora caliendrum and $S$. hystrix were found in situ in the reef around Sesoko Island in June 1998 (Fig. 1). Contacting

Table 1. Seriatopora hystrix and S. caliendrum. A summary of genetic and age combinations of coral pairs. The numbers of pairs and combinations (in brackets) with each genetic combination group are shown. For detailed information on each pair, see Fig. 4

\begin{tabular}{|c|c|c|c|c|}
\hline & \multirow{3}{*}{$\begin{array}{c}\text { Sibling } \\
\text { S. hystrix }\end{array}$} & \multirow{2}{*}{\multicolumn{2}{|c|}{$\begin{array}{l}\text { - Allografts } \\
\text { Non sibling }\end{array}$}} & \multirow{3}{*}{$\begin{array}{c}\text { Xenografts } \\
\text { S. caliendrum } \\
\text { and } S \text {. hystrix }\end{array}$} \\
\hline & & & & \\
\hline & & S. caliendrum & S. hystrix & \\
\hline Same age & $4(1)$ & $3(1)$ & $15(3)$ & $17(4)$ \\
\hline Different age & 0 & $9(5)$ & $1(1)$ & $9(6)$ \\
\hline Total & $4(1)$ & $12(6)$ & $16(4)$ & $26(10)$ \\
\hline
\end{tabular}

branch pairs of the 2 species were carefully removed with forceps and examined for their contact reactions under a dissecting microscope in order to ascertain the nature of the response in adult colonies.

\section{RESULTS}

\section{Contact reactions of juvenile Seriatopora caliendrum and S. hystrix}

Three tissue contact reactions were observed in juvenile coral pairs of Seriatopora caliendrum and S. hystrix among all combination groups of different genetic distances: fusion, nonfusion and incompatible fusion (after Hidaka et al. 1997). In the nonfusion reaction, although tissues of paired colonies were directly in contact during the initial stages of the reaction, they maintained clear tissue separation. Within a few weeks, a thin skeletal wall was formed vertically at the interface of these pairs, physically separating the tissues of the colonies (Fig. 2A). In the fusion reaction, tissues and skeletons of paired colonies formed a continuum and zooxanthellae were uniformly distributed across the original contact area of the genets comprising the chimera (Fig. 2B). In the incompatible fusion reaction, tissues and skeletons of paired colonies appeared to be fused but the colonies were separated by a distinct white border zone (WBZ), which usually covered the entire interface. This 
zone contained few zooxanthellae and no polyp development was observed there throughout the experiment (Fig. 3). Tissue and skeleton of the WBZ did not appear to grow much; hence, its width became narrower relative to other parts of the colonies that continued to grow (Fig. 3A-C).

In some incompatible fusion pairs, polyp absorption was observed proximal to the WBZ. We did not detect any clear directionality in polyp absorption between the paired colonies in the small number of cases observed: 2 non sibling allografts of Seriatopora caliendrum (nos. 1 and 3 in Fig. 4) and 2 xenografts (nos. 42 and 54). The area where the absorbed polyps had resided later turned into a new white zone, resulting in expansion of the WBZ.

In 15 out of 27 incompatible fusion pairs, a skeletal rise in the WBZ was observed during the last 7 mo of the experimental period (Figs. $3 \& 4$ ). The skeleton of the WBZ continued to rise gradually, resulting in a thick skeletal wall being covered with the white zone tissue, separating the juvenile colonies in the pair (Fig. 3D, E).

Overgrowth was observed in 1 non sibling allograft pair of Seriatopora hystrix and in 8 xenograft pairs (Figs. 4 \& 5). In the latter, $S$. caliendrum colonies always overgrew $S$. hystrix colonies. Overgrowth usually occurred in pairs exhibiting nonfusion. However, in 2 xenografts (nos. 43 and 46, Fig. 4), overgrowth also occurred during incompatible fusion. In these cases, the skeletal rise in the WBZ occurred not upwards but sideways towards $S$. hystrix, resulting in its overgrowth by $S$. caliendrum. Although none of the $S$. hystrix colonies were completely overgrown, most of their basal area had been covered by $S$. caliendrum by the end of the 1 yr experiment (Fig. 5B).
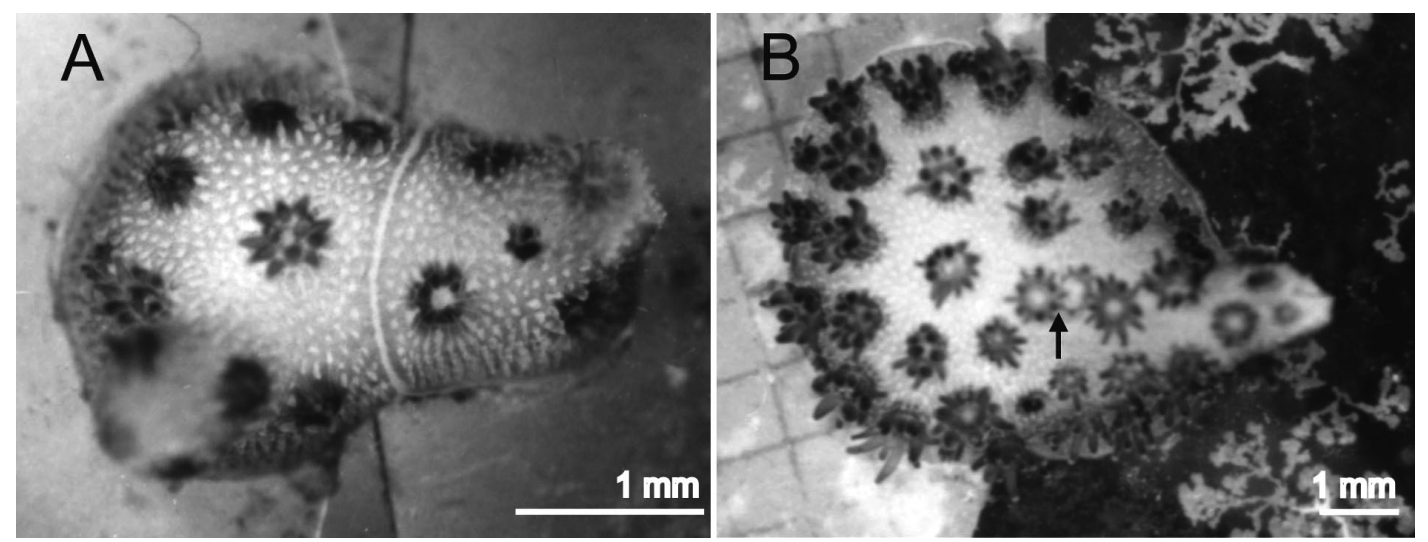

Fig. 2. Seriatopora caliendrum and S. hystrix. (A) A typical nonfusion reaction observed in a xenograft of $S$. caliendrum (left) and S. hystrix (right) on 25 September 1997, ca. 14 wk after initial contact (pair no. 32 in Fig. 4). A thin skeletal wall at the interface (white border line) separates tissues of the 2 colonies. (B) $S$. caliendrum: a typical fusion reaction observed in a non sibling allograft, Sc7B (5) (left) vs. Sc12S (1) (right), (pair no.10 in Fig. 4) on December 3 1997, ca. 19 wk after initial contact (indicated by arrow)
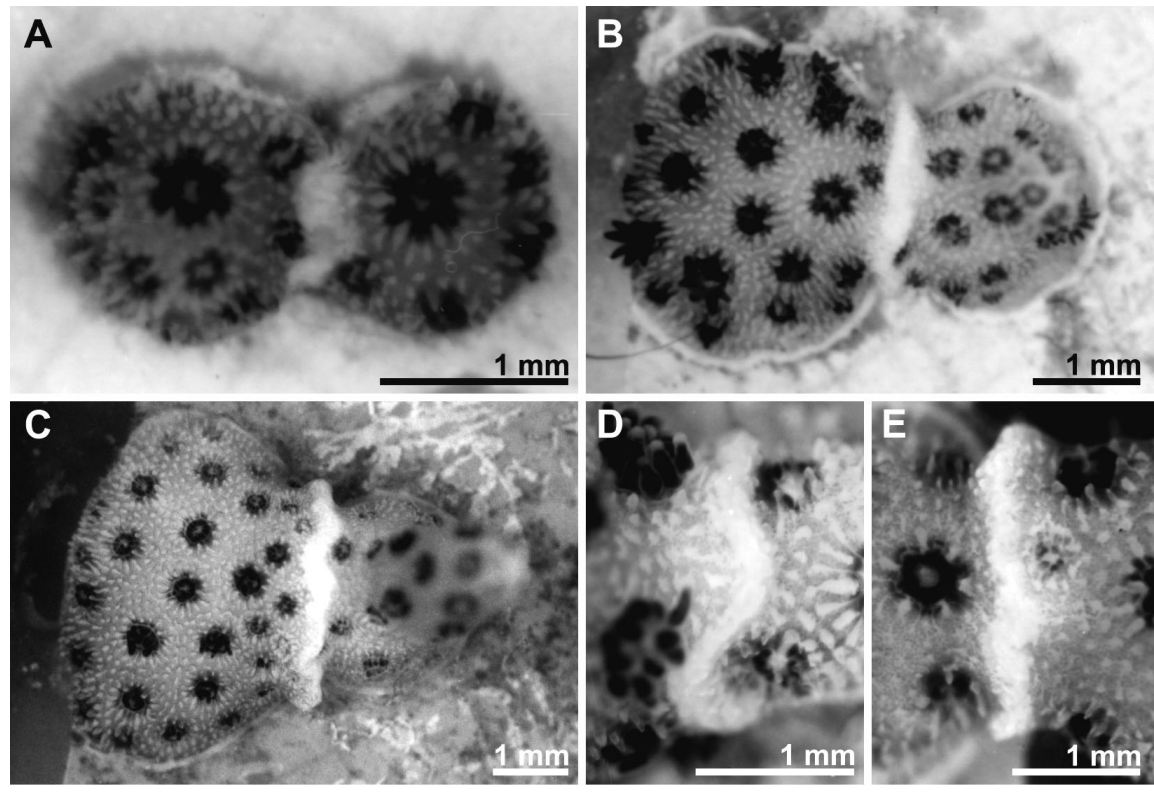

Fig. 3. Seriatopora caliendrum and S. hystrix: Incompatible fusion reaction. (A-C) A typical incompatible fusion reaction and skeletal rise of the 'white border zone' observed in a non sibling allograft of $S$. caliendrum, Sc12S(3) (left) vs. Sc15S(1) (right), (pair no. 5 in Fig. 4) on (A) October 23 1997, (B) December 18 1997 and (C) February 26 1998, approximately 12, 20 and 30 wk after initial contact, respectively. (D, E) Skeletal rises of white border zone, (D) in a xenograft of $S$. caliendrum (left) and $S$. hystrix (right) on December 18 1997, (pair no. 54 in Fig. 4) and (E) in a non sibling allograft of $S$. caliendrum on December 31997 (pair no. 11 in Fig. 4), Sc7B (5) (left) vs. Sc12S (1) (right), approximately $17 \mathrm{wk}$ after the initial contact, respectively 


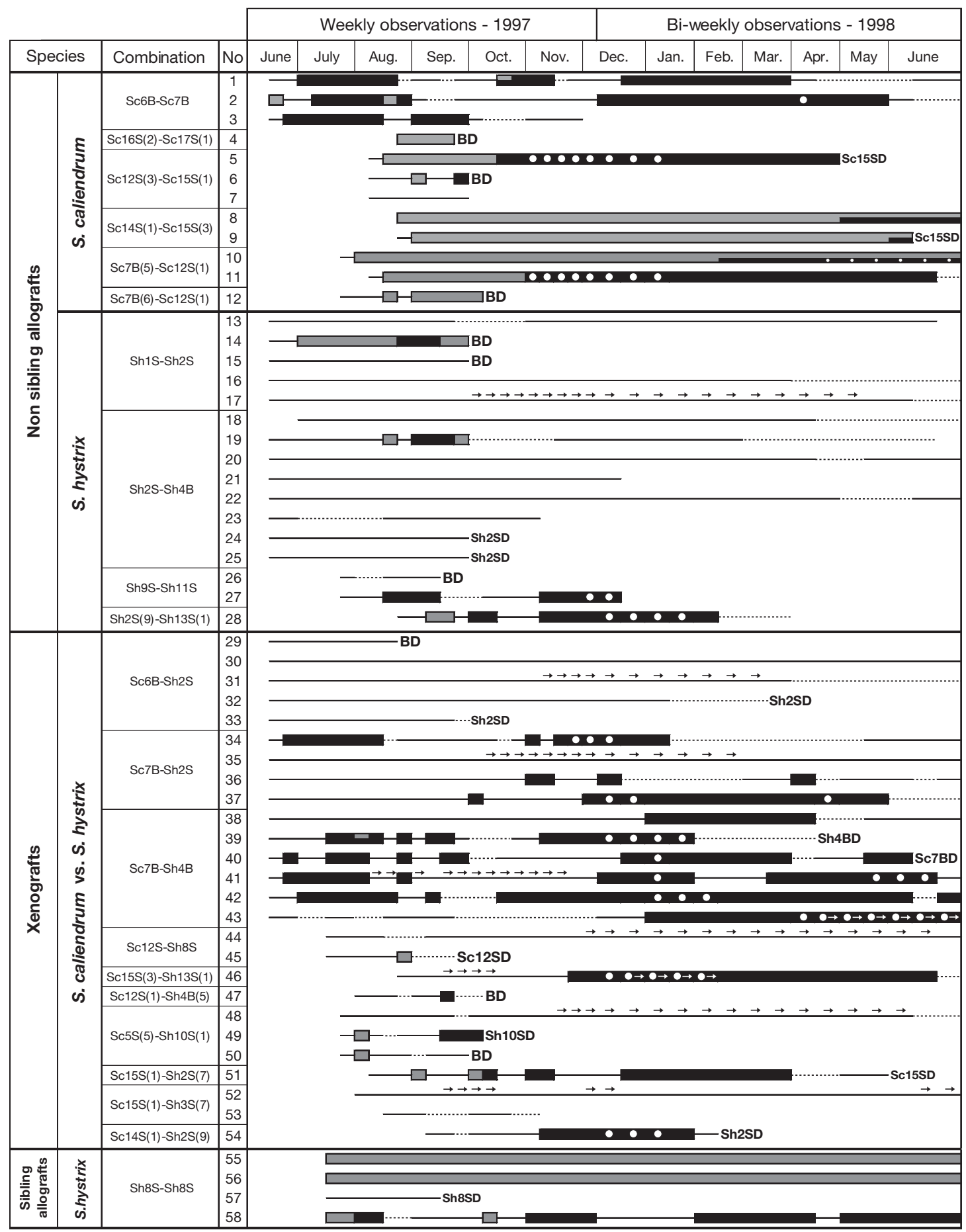

Fig. 4. Seriatopora caliendrum and S. hystrix. Dynamics of contact reactions in juvenile coral pairs. Horizontal lines indicate changes in the contact reaction of each grafted pair throughout the experiment. The beginning and end of each line indicates the dates on which the first and last observations were conducted. Weekly observations were carried out for the first 6 mo and fortnightly for the next 6 mo. The combination column provides details of each colony in a pair as follows: species name (Sc, $S$. caliendrum $_{i}$ Sh, S. hystrix), maternal colony no., collection site (S, Sesoko; B, Bise) and juvenile colony age categorized within the 1st to 9th wk after planulation at initial contact (in brackets). Colonies without brackets are those brought into contact within the first $24 \mathrm{~h}$ after planulation. Within each combination group, paired colonies were numbered consecutively, so that a higher combination serial number denoted a pair with a larger age difference between the grafted coral juveniles. Horizontal lines discontinuing before the end of the experiment (June 1998) indicate that either one of the grafted juvenile colonies died (D), or both colonies died (BD). In cases where the juvenile colonies detached and fell off the plastic sheets, there is no notation at the end. Symbols on each horizontal line are as follows: nonfusion (thin continuous line); disconnection (thin broken line); fusion (grey thick line); incompatible fusion (black thick line); skeletal rise of white border zone (open circle in black thick line); overgrowth (arrows pointing out the direction of overgrowth, e.g. in pair no.17, Sh1S-Sh2S, the direction of arrows points from left to right signifying that Sh1S was overgrowing Sh2S at the indicated time). Mixed grey and black thick lines (Pairs 1, 8, 9, 10 and 39) indicate mixed reactions of fusion and incompatible fusion 


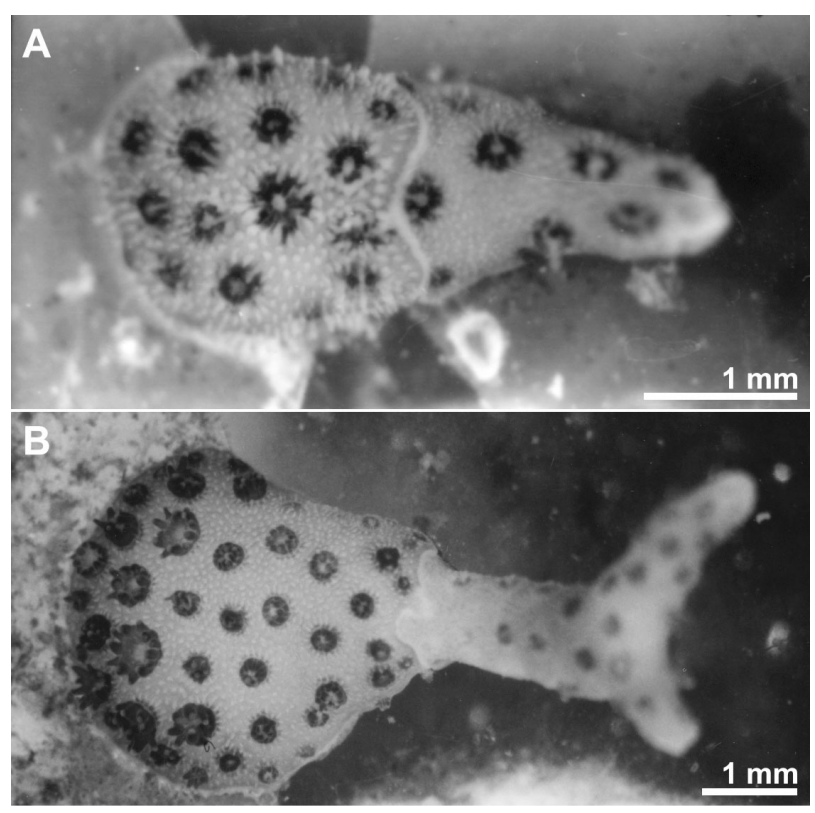

Fig. 5. Seriatopora caliendrum and S. hystrix. An overgrowth observed in a xenograft of $S$. caliendrum (left) and $S$. hystrix (right) (pair no. 35 in Fig. 4) on (A) October 231997 and (B) February 25 1998, approximately 18 and 36 wk after initial contact, respectively. Note differences in the initial growth strategy between the 2 species: $S$. caliendrum starts off by horizontal growth, while $S$. hystrix starts to grow vertically

In 22 pairs of all combination groups (1 sibling allograft of Seriatopora hystrix, 5 non sibling allografts of S. caliendrum, 5 non sibling allografts of S. hystrix and 11 xenografts), one or both colonies died during the experimental period. No obvious tendency was detected with regard to the identity of the dead partner within the pairs (Fig. 4).

Finding naturally occurring adult xenograft pairs of Seriatopora caliendrum and S. hystrix colonies in the field (Fig. 1) gave us an opportunity to compare the outcome of contact reactions in adult coral xenografts to that of juvenile xenografts. Among a total of 80 contacting branch-pairs inspected in the xenograft colonypairs, the vast majority of contact reactions were nonfusion $(87.5 \%)$, with incompatible fusion reactions comprising only $2.5 \%$ and disconnection (separation between tissues of a contacting branch pair) $10 \%$.

\section{Transition of contact reactions in juvenile Seriatopora caliendrum and S. hystrix}

Fusion reactions were observed 27 times in 21 pairs (recording either 1 or 2 occurrences per pair) in all genetic combination groups during the experimental period (see Fig. 4). Among 21 contact reactions observed prior to the occurrence of the fusion reactions (i.e. nonfusion or incompatible fusion), $81 \%$ were nonfusion (Fig. 4). The onset of fusion reaction was observed only between grafted colonies younger than 4 mo post settlement. Except for 2 sibling allografts of Seriatopora hystrix (nos. 55 and 56 in Fig. 4) and 3 non sibling allografts of $S$. caliendrum (nos. 8, 9 and 10), all fusion reactions eventually transformed into other contact reactions (nonfusion or incompatible fusion) or disconnection (separation between tissues of paired colonies by necrosis of the border tissue at the interface) within that period. Although the 3 allografts of $S$. caliendrum maintained fusion reaction throughout almost the entire experimental period, the reactions partially transformed into incompatible fusions 1 to 5 mo before the end of the experiment. Hence, except for the 2 sibling $S$. hystrix allografts, no allografts or xenografts maintained stable fusion reactions at the end of the 1 yr experimental period. Among 20 contact reactions following the fusion reactions, 55\% were incompatible fusion and $45 \%$ were nonfusion reactions (Fig. 4).

The initial appearance of incompatible fusion in each grafted pair varied greatly from $2 \mathrm{wk}$ (nos. 3, 34, 40 and 41 in Fig. 4) to 41 wk (no. 9) after initial contact. During the experiment, most pairs that had exhibited an incompatible fusion reaction once, continued to exhibit this reaction repeatedly, resulting in a total of 60 occurrences in 28 pairs of all genetic combination groups (see Fig. 4). Among 58 contact reactions observed prior to the occurrence of the incompatible fusion reactions (i.e. fusion or nonfusion), most of them (81\%) were nonfusion reactions. Among 29 contact reactions observed following incompatible fusions, most of them $(86 \%)$ were nonfusion reactions. Apart from contact reactions, disconnection was observed in one third of the incompatible fusions at the end of the reaction (20 cases in the 60 incompatible fusions).

\section{Determinants of contact reactions in juvenile Seriatopora caliendrum and S. hystrix}

There were apparent differences in the incidence and duration of fusion and incompatible fusion reactions among the 4 genetic combination groups (Fig. 4). In sibling allografts of Seriatopora hystrix, $(n=4) 3$ of the 4 pairs exhibited fusion and/or incompatible fusion reactions. Two pairs (nos. 55 and 56, see Fig. 4) exhibited fusion reactions, forming a stable chimera that lasted throughout the $1 \mathrm{yr}$ experimental period. In non sibling allografts of $S$. caliendrum $(\mathrm{n}=12)$, most pairs $(92 \%)$ exhibited relatively stable fusion and/or incompatible fusion reactions, whereas in non sibling allografts of $S$. hystrix (n = 16), only $25 \%$ exhibited those reactions. In xenografts of the 2 species $(n=26), 62 \%$ 
showed fusion and/or incompatible fusion reactions. However, only 5 pairs exhibited fusion reactions that lasted for less than $2 \mathrm{wk}$, in contrast to many incompatible fusion reactions in xenografts that lasted for several months (Fig. 4).

Within various age combinations of juveniles (within the first week post settlement) and 'older' colony pairs (from the 2nd to the 9th week post settlement) examined (Fig. 4), there was no obvious effect of colony age on contact reactions of juvenile colonies. In 9 non sibling allografts of Seriatopora caliendrum (nos. 4 to 12) and 9 xenografts (nos. 46 to 54), the initial type of contact reactions and their dynamics within each genetic combination group did not appear to change with increasing pair age differences, but rather remained similar within each genetic combination group.

\section{DISCUSSION}

Allografts of juvenile scleractinian corals of Seriatopora caliendrum and $S$. hystrix displayed the same 3 contact reactions (fusion, nonfusion and incompatible fusion), as observed in those of Pocillopora damicornis (Hidaka et al. 1997) and Stylophora pistillata (Frank et al. 1997). Xenografts of juvenile corals of $S$. hystrix and $S$. caliendrum also showed the same 3 contact reactions as the allografts. Therefore, these 3 reactions may be considered general contact responses in grafts of genetically different juvenile Pocilloporid corals.

The incidences of the 3 contact reactions differed greatly between non sibling allografts of Seriatopora caliendrum and those of S. hystrix (Fig. 4). Most non sibling allograft pairs of $S$. caliendrum had fusion and/or incompatible fusion reactions, while only a few non sibling allograft pairs of $S$. hystrix had these reactions. The primary cause for these differences may be attributed to differences in the general growth direction of the 2 species observed in grafted pairs. While juvenile colonies of $S$. caliendrum exhibited preferential horizontal growth, those of $S$. hystrix tended to grow vertically (Fig. 5). These differential growth strategies may have caused $S$. caliendrum to come into contact with partner-colonies more frequently than $S$. hystrix, resulting in more occurrences of fusion and incompatible fusion reactions. In xenografts, overgrowth was always unidirectional, with $S$. caliendrum overgrowing $S$. hystrix. Hence, we conclude that there is a general hierarchical relationship between juveniles of the 2 species, in which $S$. caliendrum outcompetes $S$. hystrix, a result that we have also recorded in situ in all the naturally occurring xenografts of the 2 species. This outcome may also result from dif- ferences in the growth strategies of juvenile colonies of the 2 species.

In order to examine possible effects of genetic distance on contact reactions of juvenile corals, we examined contact reactions not only between non sibling allografts of Seriatopora caliendrum and S. hystrix, but also between xenografts of the 2 species. In non sibling allografts of $S$. caliendrum, fusion and incompatible fusion were the 2 dominant and relatively stable reactions (Fig. 4). In non sibling allografts of S. hystrix, however, the 2 contact reactions were rare, probably stemming from the initial vertical growth direction of $S$. hystrix discussed above. In contrast, in the xenograft pairs, fusion reactions were rarely observed and when they did occur, they lasted only a short period ( $<2 \mathrm{wk})$. Instead, nonfusion reactions were the dominant reaction, along with incompatible fusion reactions, both of which could already be observed at early contact stages. The fusion reaction is considered a histocompatible reaction, whereas incompatible fusion is regarded as a histoincompatible reaction, which eventually transforms into nonfusion or disconnection, via tissue necrosis of the WBZ (Frank et al. 1997, Hidaka et al. 1997). These results suggest a possibility that the allorecognition system already functions to some extent even in juvenile corals, since it appears to identify genetically more distant encounters better than less distant encounters. In addition, contact reactions were largely consistent between pair replicates of each combination and within each of the 4 genetic combination groups, as also noted by Hidaka et al. (1997). We, therefore, conclude that, in juvenile corals, the genetic combination of each graft is a primary determinant of the contact reaction and its endurance.

Obviously, colony age is another important factor to be considered in contact reactions, since fusion and incompatible fusion were rarely observed in allografts and xenografts of adult scleractinian corals, whereas these are 2 of the 3 common contact reactions observed so far in juvenile scleractinian corals. These 2 reactions were suggested to occur in allografts of juvenile scleractinian corals because they still lack a fully developed allorecognition system (Hidaka 1985, Frank et al. 1997, Hidaka et al. 1997). Frank et al. (1997) reported that contact reactions of allografts of juvenile Stylophora pistillata corals were fully dependent on the age, or maturation stage of the allorecognition system. In the present study, we did not detect any apparent age effect on contact reactions in 10 non sibling allografts and 9 xenografts of various age combinations paired from juvenile Seriatopora caliendrum and S. hystrix colonies aged 0 to $59 \mathrm{~d}$ post settlement. Hidaka et al. (1997) also reported that the age of grafted colonies did not appear to affect contact reactions in allografts of Pocillopora damicornis aged between $7 \mathrm{~d}$ and $3 \mathrm{mo}$ 




Fig. 6. Seriatopora caliendrum and S. hystrix: Schematic diagram signifying general transition-patterns of contact reactions observed in allogeneic and xenogeneic juvenile Seriatopora coral-pairs. Transition patterns indicated by Arrow 1 occurred in low frequency in genetically distant coral pairs and disappeared approximately 4 mo after settlement of either grafted colonies. Transition patterns indicated by Arrow 2 may also disappear as the fully functional allorecognition system matures. Hence, transition patterns indicated by Arrow 3 will

develop eventually in contact reactions of adult corals

post planulation. There is, however, similarity between the 3 studies (i.e. Frank et al. 1997, Hidaka et al. 1997 and the present study). In all 3 studies, the onset of fusion reactions in allografts and xenografts occurred only when grafted colonies were younger than 4 mo post settlement. In the present study, however, we also found that most of the fusion reactions transformed into other contact reactions (i.e. nonfusion or incompat- ible fusion reactions) or disconnection within that period. The results of the present study are consistent with those of Barki et al. (2002) on allogenic fusions of primary polyps in 4 soft coral species. In that study, most of the fusions occurred within less than 1 mo post settlement and most of those fusions resulted in separation or death of the chimera partners within the first 2 mo after fusion.

Our results suggest that a 'basic allorecognition system' (i.e. not fully mature or fully functional) has already developed in the juvenile Seriatopora corals within the first 4 mo post settlement. Complete maturation of the allorecognition system, found in adult corals, is likely to require more time, since incompatible fusion reactions still appeared, even 1 yr later, in most of the allograft and xenograft pairs in our study. However, as the functional allorecognition system matures, the incompatible fusion may eventually disappear, as observed in contact reactions of naturally occurring xenografts of adult colonies (Fig. 1). Indeed, studies on the colonial hydroid Hydractinia symbiolongicarpus showed that juveniles of that species fused to parental strains but once they achieved reproductive maturity, most of the fusion reactions transformed into disconnection by tissue necrosis at the border line (Shenk \& Buss 1991).

Fig. 6 summarizes general transition patterns of contact reactions observed in the present study in allografts and xenografts of juvenile Seriatopora corals. It is apparent from Fig. 6 that, by affecting both onset period and duration of the fusion (i.e. within the first 4 mo after settlement) and incompatible fusion reactions (i.e. within the juvenile stage), it is the developmental stage of the allorecognition system that determines the type and fate of contact reactions in juvenile corals. Fuchs et al. (2002) also reported that the onset period and fates of allogenic fusions in a colonial hydroid, Hydractinia were determined by the developmental stage of the allorecognition system. In conclusion, the developmental stage of the allorecognition system in corals appears to constitute a significant determinant of contact reactions in juvenile corals.

Acknowledgements. This work was supported by a fellowship to Y.L. from the Japanese Ministry of Science, Education, Culture and Sports (Foreign Visiting Professor) at the Tropical Biosphere Research Center-Sesoko Station, University of the Ryukyus. We thank the former Director of the Station Dr. K. Takano and the present Director Prof. M. Murai for providing the opportunity and excellent facilities to carry out the research. The invaluable assistance and kind hospitality of the staff of Sesoko Station is greatly appreciated, especially that of Dr. K. Sakai, Dr. A. Takemura, Mr. Y. Nakano, Mrs. C. Uchima and Mr. S. Nakamura. We are particularly grateful to Prof. M. Hidaka and Drs. U. Frank, O. Mokady, E. KramarskyWinter and 2 anonymous referees for their helpful comments on the manuscript. 


\section{LITERATURE CITED}

Barki Y, Gateño D, Graur D, Rinkevich B (2002) Soft-coral natural chimerism: a window in ontogeny allows the creation of entities comprised of incongruous parts. Mar Ecol Prog Ser 231:91-99

Chadwick-Furman N, Rinkevich B (1994) A complex allorecognition system in a reef-building coral: delayed responses, reversals and nontransitive hierarchies. Coral Reefs 13:57-63

Chornesky EA (1991) The ties that bind: inter-clonal cooperation may help a fragile coral dominate shallow highenergy reefs. Mar Biol 109:41-51

Frank U, Rinkevich B (1994) Nontransitive patterns of historecognition phenomena in the Red Sea hydrocoral Millepora dichotoma. Mar Biol 118:723-729

Frank U, Bak RPM, Rinkevich B (1996) Allorecognition responses in the soft coral Parerythropodium fulvum fulvum from the Red Sea. J Exp Mar Biol Ecol 197:191-201

Frank U, Oren U, Loya Y, Rinkevich B (1997) Alloimmune maturation in the coral Stylophora pistillata is achieved through three distinctive stages, 4 months post-metamorphosis. Proc R Soc Lond B 264:99-104

Fuchs MA, Mokady O, Frank U (2002) The ontogeny of allorecognition in a colonial hydroid and the fate of

Editorial responsibility: Otto Kinne (Editor-in-Chief), Oldendorf/Luhe, Germany early established chimeras. Int J Dev Biol 46:699-704 Grosberg RK (1988) The evolution of allorecognition specificity in clonal invertebrates. Q Rev Biol 63:377-412

Hidaka M (1985) Tissue compatibility between colonies and between newly settled larvae of Pocillopora damicornis. Coral Reefs 4:111-116

Hidaka M, Yurugi K, Sunagawa S, Kinzie RA (1997) Contact reactions between young colonies of the coral Pocillopora damicornis. Coral Reefs 16:13-20

Jokiel PL, Bigger CH (1994) Aspects of histocompatibility and regeneration in the solitary reef coral Fungia scutaria. Biol Bull (Woods Hole) 186:72-80

Leddy SV, Green DR (1991) Historecognition in the Cnidaria. In: Warr GW, Cohen N (eds) Phylogenesis of immune functions. CRC Press, Boca Raton, FL, p 103-116

Permata WD, Kinzie RA III, Hidaka M (2000) Histological studies on the origin of planulae of the coral Pocillopora damicornis. Mar Ecol Prog Ser 200:191-200

Rinkevich B, Frank U, Bak RPM, Müller WEG (1994) Alloimmune responses between Acropora hemprichi conspecifics: nontransitive patterns of overgrowth and delayed cytotoxicity. Mar Biol 118:731-737

Shenk MA, Buss LW (1991) Ontogenetic changes in fusibility in the colonial hydroid Hydractinia symbiolongicarpus. J Exp Zool 257:80-86

Submitted: December 1, 2003; Accepted: August 3, 2004 Proofs received from author(s): January 25, 2005 